\title{
HOMOTOPICAL ASPECTS OF LIE ALGEBRAS
}

\author{
GRAHAM J. ELLIS
}

(Received 5 July 1991)

Communicated by R. H. Street

\begin{abstract}
The Hurewicz theorem, Mayer-Vietoris sequence, and Whitehead's certain exact sequence are proved for simplicial Lie algebras. These results are applied, using crossed module techniques, to obtain information on the low dimensional homology of a Lie algebra, and information on aspherical presentations of Lie algebras.
\end{abstract}

1991 Mathematics subject classification (Amer. Math. Soc.): 18 G 55.

\section{Introduction}

Many results in the homology of groups are best proved using techniques from the homotopy theory of topological spaces. Analogous results in the homology of Lie algebras rely on different proofs because of an apparent lack of a suitable homotopy theory. However, it is well known that (connected CW-) topological spaces are equivalent (up to homotopy) to simplicial groups. In the light of this equivalence, and in the light of the close analogy between general algebraic properties of groups and Lie algebras, this article investigates the homotopy theory of simplicial Lie algebras with a view to homological applications.

In Section 1 we recall from [6,16 and 8] some general results on simplicial objects and homotopical algebra. In particular, for a simplicial object in an algebraic category such as the category of Lie algebras, we recall suitable definitions of homotopy groups and homology groups. Although in subsequent sections we have chosen to work solely with Lie algebras, much of the theory

(C) 1993 Australian Mathematical Society $0263-6115 / 93 \$ A 2.00+0.00$ 
carries over to other algebraic settings such as commutative algebras, Jordan algebras, and associative algebras. Indeed, all of the theory carries over to the category of groups, in the main yielding new proofs of known results.

In Section 2 we analyse the low dimensional parts of the Moore complex of a simplicial Lie algebra. We recall from [14] that a Moore complex of length one is equivalent to a crossed module. We then capture the structure of a Moore complex of length 2 in the definition of a 2-crossed module; this is the Lie algebraic version of a group theoretic notion defined in [5].

In Section 3 we consider free crossed modules and free 2-crossed modules. A group theoretic version of Theorem 7 of this section was proved topologically in [1]; subsequently, as the main application of the paper [2], an algebraic proof was given. Our proof of Theorem 7 converts to a new algebraic proof in the group case.

In Section 4 we prove Lie algebraic versions of some classical results including the Hurewicz Theorem, Whitehead's Certain Exact Sequence, and the Mayer-Vietoris sequence.

In Section 5 we give a description of coproducts of crossed modules, copying group theoretic ideas from [4].

In Section 6 and 7 we apply our results to the Eilenberg-MacLane homology of Lie algebras and to the study of aspherical presentations of Lie algebras.

\section{Simplicial Algebras, Homotopy and Homology}

Let $\mathscr{C}$ be an algebraic category such as the category of Lie algebras, groups, commutative algebras, associative algebras or Jordan algebras. We shall refer to the objects of $\mathscr{C}$ as algebras, and to the maps as homomorphisms. The kernel of a homomorphism will be referred to as an ideal. We let 0 denote the null object in $\mathscr{C}$. There is a forgetful functor $U: \mathscr{C} \rightarrow \mathscr{S e t}$ from $\mathscr{C}$ to the category of sets, which has a left adjoint $F: \operatorname{Set} \rightarrow \mathscr{C}$. We shall say that an algebra is free if it is of the form $G=F(X)$ for some set $X$.

A simplicial algebra is a sequence of algebras $\mathbf{G}=\left\{G_{0}, G_{1}, \ldots, G_{n}, \ldots\right\}$ together with homomorphisms

$$
\begin{aligned}
& d_{i}: G_{n} \rightarrow G_{n-1}, \\
& s_{i}: G_{n} \rightarrow G_{n+1},
\end{aligned}
$$

for each $0 \leq i \leq n$. These homomorphisms are required to satisfy the 
simplicial identities

$$
\begin{aligned}
& d_{i} d_{j}=d_{j-1} d_{i} \quad \text { for } i<j, \\
& d_{i} s_{i}= \begin{cases}s_{j-1} d_{i} & \text { for } i<j, \\
\text { identity } & \text { for } i=j, j+1, \\
s_{j} d_{i-1} & \text { for } i>j+1,\end{cases} \\
& s_{i} s_{j}=s_{j+1} s_{i} \quad \text { for } i \leq j .
\end{aligned}
$$

A simplicial map $f: \mathbf{G} \rightarrow \mathbf{G}^{\prime}$ is a family of homomorphisms $f_{n}: G_{n} \rightarrow G_{n}^{\prime}$ commuting with the $d_{i}$ and $s_{i}$. We let $s \mathscr{C}$ denote the category of simplicial algebras.

The Moore complex of a simplicial algebra $\mathbf{G}$ is the complex

$$
M(\mathbf{G}) \quad \cdots \rightarrow M_{n} \stackrel{\partial_{n}}{\longrightarrow} M_{n-1} \rightarrow \cdots \rightarrow M_{1} \stackrel{\partial_{1}}{\longrightarrow} M_{0} \rightarrow 0
$$

where

$$
\begin{aligned}
& M_{0}=G_{0}, \\
& M_{n}=\bigcap_{i \neq n} \operatorname{ker}\left(d_{i}: G_{n} \rightarrow G_{n-1}\right), \\
& \partial_{n}=d_{n}\left(\text { restricted to } M_{n}\right) .
\end{aligned}
$$

The simplicial identities imply that $\partial_{n}\left(M_{n}\right)$ is an ideal in $\operatorname{ker}\left(\partial_{n-1}\right)$. The homotopy groups of a simplicial algebra $\mathbf{G}$ are defined as the homology groups of the Moore complex $M(G)$ :

$$
\pi_{n}(\mathbf{G})=\operatorname{ker}\left(\partial_{n}: M_{n} \rightarrow M_{n-1}\right) / \operatorname{im}\left(\partial_{n+1}: M_{n+1} \rightarrow M_{n}\right), \quad n \geq 0 .
$$

A simplicial map $f: \mathbf{G} \rightarrow \mathbf{G}^{\prime}$ is called a weak equivalence if it induces isomorphisms $\pi_{n}(\mathbf{G}) \cong \pi_{n}\left(\mathbf{G}^{\prime}\right)$ for $n \geq 0$. A simplicial map $f: \mathbf{G} \rightarrow \mathbf{G}^{\prime}$ is called a fibration if $f_{n}: G_{n} \rightarrow G_{n}^{\prime}$ is surjective for $n \geq 0$. A simplicial map $f: \mathbf{G} \rightarrow \mathbf{G}^{\prime}$ is called a cofibration if it can be factored as $f=p i$ with $p: \mathbf{Z} \rightarrow \mathbf{G}^{\prime}$ both a fibration and weak equivalence, and $i: \mathbf{G} \rightarrow \mathbf{Z}$ a free map in the following sense: there are subsets $X_{n} \subseteq Z_{n}$ satisfying

(i) the $X_{n}$ are stable under all degeneracy operators (that is, if $x \in X_{n}$ then $s_{i} x \in X_{n+1}$ ),

(ii) $Z_{n}$ is isomorphic to the coproduct $G_{n} \vee F X_{n}$ where $F X_{n}$ is the free algebra on $X_{n}$, the isomorphism being $i_{n}+g_{n}: G_{n} \vee F X_{n} \rightarrow Z_{n}$ with $g_{n}$ the unique homomorphism extending the identity on $X_{n}$. With these notions of weak equivalence, fibration and cofibration the category of simplicial algebras is a closed model category in the sense of Quillen [16, Theorem II.4.4]. A simplicial algebra $\mathbf{G}$ is said to be cofibrant or free if $G_{n}$ is a free object in $\mathscr{C}$ for all $n \geq 0$, and if the free bases are stable under all degeneracy operators (that is, if $x \in G_{n}$ is a basis element then so is $s_{i} x$ ). (In the terminology 
of [16] all simplicial algebras are fibrant.) We let $s \mathscr{E}_{0}$ denote the category of free simplicial algebras. We let $H o(s \mathscr{C})$ denote the category of simplicial algebras localised with respect to weak equivalences. (In other words, there is a functor $\gamma: s \mathscr{C} \rightarrow H o(s \mathscr{C})$ having the following universal property: $\gamma$ sends weak equivalences to isomorphisms; any other functor $s \mathscr{C} \rightarrow \mathscr{D}$ which maps weak equivalences to isomorphisms factors through $\gamma$.)

If $f, g: \mathbf{G} \rightarrow \mathbf{G}^{\prime}$ are two simplicial maps, a homotopy of $f$ onto $g$ is a family of homomorphisms

$$
h_{i}: G_{n} \rightarrow G_{n+1}^{\prime}, \quad 0 \leq i \leq n
$$

for each $n \geq 0$ which satisfies the following identities:

$$
\begin{gathered}
d_{0} h_{0}=f \\
d_{n+1} h_{n}=g \\
\left\{\begin{aligned}
d_{i} h_{j}=h_{j-1} d_{i} \quad i<j, \\
d_{j+1} h_{j+1}=d_{j+1} h_{j} \\
d_{i} h_{j}=h_{j} d_{i-1} \quad i>j+1, \\
s_{i} h_{j}=h_{j+1} s_{i} \quad i \leq j, \\
s_{i} h_{j}=h_{j} s_{i-1} \quad i>j .
\end{aligned}\right.
\end{gathered}
$$

Homotopy is an equivalence relation on maps between free simplicial algebras. Let $\pi\left(s \mathscr{C}_{c}\right)$ be the category whose objects are the free simplicial algebras, and whose arrows are the homotopy equivalence classes of simplicial maps. Theorem I.1 of [16] states that the inclusion functor $s \mathscr{C}_{c} \hookrightarrow s \mathscr{C}$ induces an equivalence of categories $\pi(s \mathscr{C}) \simeq H o(s \mathscr{C})$.

Suppose given an endofunctor $E: \mathscr{C} \rightarrow \mathscr{C}$, and a simplicial algebra $\mathbf{G}$. By axiom (M2) of a model category (see [16]) we can choose a free simplicial algebra $\mathbf{F}$ such that there is a weak equivalence $\mathbf{F} \stackrel{\simeq}{\rightrightarrows} \mathbf{G}$. On applying $E$ dimensionwise to $\mathrm{F}$ we obtain a simplicial algebra $E \mathrm{~F}=\left\{E F_{0}, \ldots, E F_{n}, \ldots\right\}$ whose homotopy groups we denote by

$$
D_{n}^{E}(\mathbf{G})=\pi_{n}(E \mathbf{F}), \quad n \geq 0 .
$$

These homotopy groups do not depend on the choice of $\mathbf{F}$. To see this suppose that $\mathbf{F}$ and $\mathbf{F}^{\prime}$ are two free simplicial algebras, both weakly homotopic to $\mathbf{G}$. Then the images of $\mathbf{F}$ and $\mathbf{F}^{\prime}$ in $H o(s \mathscr{C})$ are isomorphic. From the equivalence $\pi\left(s \mathscr{C}_{c}\right) \simeq H o(s \mathscr{C})$ it follows that the images of $F$ and $F^{\prime}$ in $\pi\left(s \mathscr{E}_{c}\right)$ are isomorphic. Since the endofunctor $E$ preserves homotopies, we see that $E \mathbf{F}$ is homotopy equivalent to $E \mathbf{F}^{\prime}$, and hence that the homotopy groups of $E \mathbf{F}$ are isomorphic to those of $E \mathbf{F}^{\prime}$.

Let $\mathscr{C}^{\text {ab }}$ be the category of abelian group objects in $\mathscr{C}$. Thus if $\mathscr{C}$ is the category of groups then $\mathscr{C}^{\mathrm{ab}}$ is the category of abelian groups. If $\mathscr{C}$ is any 
of the categories of Lie algebras, commutative algebras, associative algebras or Jordan algebras over a fixed ground ring $\Lambda$, then $\mathscr{C}^{\text {ab }}$ is the category of $\Lambda$-modules. There is a functor $(-)^{\text {ab }}: \mathscr{C} \rightarrow \mathscr{C}^{\text {ab }}$ which is left adjoint to the inclusion $\mathscr{C}^{\mathrm{ab}} \rightarrow \mathscr{C}$.

We define the homology groups of a simplicial algebra $\mathbf{G}$ to be

$$
H_{n}(\mathbf{G})=D_{n}^{E}(\mathbf{G}) \text { with } E=(-)^{\text {ab }}, \quad \text { for } n \geq 0 \text {. }
$$

By a $k$-truncated simplicial algebra we mean a collection of algebras $\left\{G_{0}\right.$, $\left.\ldots, G_{k}\right\}$ and homomorphisms $d_{i}: G_{n} \rightarrow G_{n-1}$ for $0 \leq i \leq n, 0 \leq n \leq k$ and $s_{i}: G_{n} \rightarrow G_{n+1}$ for $0 \leq i \leq n, 0 \leq n \leq k$ which satisfy the simplicial identities. Clearly by forgetting higher dimensions, any simplicial algebra $\mathbf{G}$ yields a $k$-truncated simplicial algebra $\operatorname{tr}^{k} \mathbf{G}$. The functor $\operatorname{tr}^{k}$ admits a right adjoint $\operatorname{cosk}^{k}$, called the $k$-coskeleton functor, and a left adjoint $\mathrm{sk}^{k}$ called the $k$-skeleton functor. We recall from [8] a brief description of these functors.

Suppose $\operatorname{tr}^{k}(\mathbf{G})=\left\{G_{0}, \ldots, G_{k}\right\}$ is a $k$-truncated simplicial algebra. A family of homomorphisms

$$
\left(\delta_{0}, \ldots, \delta_{k+1}\right): X_{k+1} \underset{\delta_{0}}{\stackrel{\delta_{k+1}}{:}} G_{k}
$$

is the simplicial kernel of the family of boundary homomorphisms $\left(d_{0}, \ldots\right.$, $\left.d_{k}\right)$ if it has the following universal property: given any family $\left(d_{0}, \ldots\right.$, $d_{k+1}$ ) of $k+2$ homomorphisms $d_{i}: Y \rightarrow G_{k}$ satisfying the identities $d_{i} d_{j}=$ $d_{j-1} d_{i}(0 \leq i<j \leq k+1)$ with the last part of the truncated simplicial algebra, there exists a unique homomorphism $f: Y \rightarrow X_{k+1}$ such that $\delta_{i} f=d_{i}$. Given the simplicial kernel $X_{k+1}$ the family of homomorphisms $\left(\alpha_{n+1, j}, \ldots, \alpha_{1 j}, \alpha_{0 j}\right)$ defined by

$$
\alpha_{i j}= \begin{cases}s_{j-1} d_{i} & i<j \\ i d & i=j, i=j+1 \\ s_{j} d_{i-1} & i>j+1\end{cases}
$$

satisfies the simplicial identities with the last part of the truncated simplicial algebra; hence there exists a unique $s_{j}: G_{k} \rightarrow X_{k+1}$ such that $\delta_{i} s_{j}=\alpha_{i j}$. We thus have a $(k+1)$-truncated simplicial algebra $\left\{G_{0}, \ldots, G_{k}, X_{k+1}\right\}$. By iterating this construction we get a simplicial algebra $\operatorname{cosk}^{k}\left(\operatorname{tr}^{k}(\mathbf{G})\right)=$ $\left\{G_{0}, \ldots, G_{k}, X_{k+1}, X_{k+2}, \ldots\right\}$ called the coskeleton of the truncated simplicial algebra. If $\mathbf{G}, \mathbf{G}^{\prime}$ are any simplicial algebras, then any truncated simplicial map $f: \operatorname{tr}^{k} \mathbf{G} \rightarrow \operatorname{tr}^{k} \mathbf{G}^{\prime}$ extends uniquely to a simplicial map $f: \mathbf{G} \rightarrow \operatorname{cosk}^{k}\left(\operatorname{tr}^{k}\left(\mathbf{G}^{\prime}\right)\right)$. 
The $k$-skeleton functor can be constructed by a dual process involving simplicial cokernels

$$
\left(s_{0}, \ldots, s_{k}\right): G_{k} \underset{s_{0}}{\stackrel{s_{k}}{\longrightarrow}} X_{k+1} .
$$

(That is, universal systems of $k+1$ arrows which satisfy $s_{i} s_{j}=s_{j+1} s_{i}$ for $0 \leq i \leq j \leq k-1$.)

\section{The Moore complex}

From this point on we restrict attention to the algebraic category of Lie algebras. Let $\Lambda$ be a commutative ring with identity. We shall use the term Lie algebra to mean a Lie algebra over $\Lambda$; we shall use $[$,$] to denote the$ Lie bracket.

We say that the Moore complex $\rightarrow M_{n} \rightarrow \cdots \rightarrow M_{1} \rightarrow M_{0}$ of a simplicial Lie algebra is of length $k$ if $M_{n}=0$ for all $n \geq k+1$ (so a Moore complex of length $k$ is also of length $r$ for $r \geq k$ ). The following lemma is a straightforward modification of Theorem 1.3 in [5].

LEMMA 1. Let $\mathbf{G}$ be a simplicial Lie algebra. The Moore complex of its $k$-coskeleton $\operatorname{cosk}^{k}\left(\operatorname{tr}^{k} \mathbf{G}\right)$ is of length $k+1$, and is identical to the Moore complex of $\mathbf{G}$ in dimensions $\leq k$. Moreover, in dimensions $k-1$ to $k+2$ the Moore complex of $\operatorname{cosk}^{k}\left(\operatorname{tr}^{k} \mathbf{G}\right)$ is an exact sequence

$$
0 \rightarrow M\left(\operatorname{cosk}^{k}\left(\operatorname{tr}^{k} \mathbf{G}\right)\right)_{k+1} \stackrel{\partial_{k+1}}{\longrightarrow} M_{k} \stackrel{\partial_{k}}{\longrightarrow} M_{k-1}
$$

where $M_{k}$ is the $k$ th term of the Moore complex of $\mathbf{G}$.

Proof. The $(k+1)$-dimensional part of $\operatorname{cosk}^{k}\left(\operatorname{tr}^{k} \mathbf{G}\right)$ can be identified with the subalgebra of the $(k+2)$-fold direct sum $G_{k}^{k+2}$ consisting of those elements $\left(x_{0}, \ldots, x_{k+1}\right)$ such that $d_{j} x_{k}=d_{k-1} x_{j}$ for $j<k$; the face maps are given by $d_{j}\left(x_{0}, \ldots, x_{k+1}\right)=x_{j}$ Thus $M\left(\operatorname{cosk}^{k}\left(\operatorname{tr}^{k} \mathbf{G}\right)\right)_{k+1}$ consists of elements $\left(0, \ldots, 0, x_{k+1}\right)$ such that $d_{j} x_{k+1}=0$ for all $j$. In other words $M\left(\operatorname{cosk}^{k}\left(\operatorname{tr}^{k} \mathbf{G}\right)\right)_{k+1}$ is the kernel of $\partial_{k}: M_{k} \rightarrow M_{k-1}$, and hence we have the exact sequence of the lemma.

The injectivity of $\partial_{k+1}$ and the isomorphism

$$
\operatorname{cosk}^{n-1}\left(\operatorname{tr}^{n-1}\left(\operatorname{cosk}^{k}\left(\operatorname{tr}^{k} \mathbf{G}\right)\right) \simeq \operatorname{cosk}^{k}\left(\operatorname{tr}^{k} \mathbf{G}\right)\right.
$$

for $n \geq k+2$ shows that the Moore complex of $\operatorname{cosk}^{k}\left(\operatorname{tr}^{k} \mathbf{G}\right)$ is of length $k+1$. 
Let $M$ and $P$ be two Lie algebras. By an action of $P$ on $M$ we mean a $\Lambda$-bilinear map $P \times M \rightarrow M,(p, m) \mapsto^{p} m$ satisfying

$$
\begin{aligned}
& {\left[p, p^{\prime}\right] } \\
& m={ }^{p}\left({ }^{p^{\prime}} m\right)-{ }^{p^{\prime}}\left({ }^{p} m\right), \\
&{ }^{p}\left[m, m^{\prime}\right]=\left[{ }^{p} m, m^{\prime}\right]+\left[m,{ }^{p} m^{\prime}\right]
\end{aligned}
$$

for all $m, m^{\prime} \in M, p, p^{\prime} \in P$.

We recall from [14] that a crossed module (in the context of Lie algebras) is a Lie homomorphism $\partial: M \rightarrow P$ together with an action of $P$ on $M$ such that

(i) $\partial\left({ }^{p} m\right)=[p,(\partial m)]$,

(ii) ${ }^{(\partial m)} m^{\prime}=\left[m, m^{\prime}\right]$,

for all $m, m^{\prime} \in M, p \in P$. A map of crossed modules $(\partial: M \rightarrow P) \rightarrow$ $\left(\partial^{\prime}: M^{\prime} \rightarrow P^{\prime}\right)$ is a pair of homomorphisms $f_{0}: P \rightarrow P^{\prime}, f_{1}: M \rightarrow M^{\prime}$ such that $f_{0} \partial=\partial^{\prime} f_{1}$ and $f_{1}\left({ }^{p} m\right)={ }^{\left(f_{0} p\right)} f_{1}(m)$ for all $m \in M, p \in P$.

The following theorem is well known, but we include its proof as subsequently we shall need to generalise it. (The proof is a slight variant of the one found in the literature.)

THEOREM 2. The category of crossed modules is equivalent to the category of simplicial Lie algebras with Moore complex of length 1.

Proof. Let $\mathbf{G}$ be a simplicial Lie algebra with Moore complex of length 1 . Put $P=G_{0}, M=\operatorname{ker}\left(d_{0}: G_{1} \rightarrow G_{0}\right)$, and $\partial=d_{1}$ (restricted to $\left.M\right)$. Then $p \in P$ acts on $m \in M$ by ${ }^{p} m=\left[s_{0} p, m\right]$, and $\partial\left({ }^{p} m\right)=\left[d_{1} s_{0} p, d_{1} m\right]=$ $[p, \partial m]$. Since the Moore complex $\cdots \rightarrow 0 \rightarrow M \stackrel{\partial}{\rightarrow} P \rightarrow 0$ is of length 1 we have

$$
\begin{aligned}
{ }^{(\partial m)} m^{\prime} & =\left[s_{0} d_{1} m, m^{\prime}\right] \\
& =\left[s_{0} d_{1} m-m, m^{\prime}\right]+\left[m, m^{\prime}\right] \\
& =d_{2}\left[\left(s_{0} m-s_{1} m\right), s_{1} m^{\prime}\right]+\left[m, m^{\prime}\right] \\
& =\left[m, m^{\prime}\right]
\end{aligned}
$$

for $m, m^{\prime} \in M$ because $\left[\left(s_{0} m-s_{1} m\right), s_{1} m^{\prime}\right]$ lies in $\operatorname{ker}\left(d_{0}\right) \cap \operatorname{ker}\left(d_{1}\right)=0$. Thus $\partial: M \rightarrow P$ is a crossed module.

Conversely, suppose we start with a crossed module $\partial: M \rightarrow P$. Using the action of $P$ on $M$ we can form the semi-direct product $M \ominus P=$ 
$\{(m, p): m \in M, p \in P\}$, in which scalar multiplication is $\lambda(m, p)=$ $(\lambda m, \lambda p)$, addition is $(m, p)+\left(m^{\prime}, p^{\prime}\right)=\left(m+m^{\prime}, p+p^{\prime}\right)$, and multiplication is $\left[(m, p),\left(m^{\prime}, p^{\prime}\right)\right]=\left(\left[m, m^{\prime}\right]-{ }^{p^{\prime}} m+{ }^{p} m^{\prime},\left[m, m^{\prime}\right]\right)$ for $\lambda \in \lambda$, $m, m^{\prime} \in M, p, p^{\prime} \in P$. There are homomorphisms

$$
\begin{array}{ll}
d_{0}: M \ominus P \rightarrow P, & (m, p) \mapsto p, \\
d_{1}: M \ominus P \rightarrow P, & (m, p) \mapsto(\partial m)+p, \\
s_{0}: P \rightarrow M \ominus P, & p \mapsto(0, p) .
\end{array}
$$

Let $G_{0}=P, G_{1}=M \ominus P$. We have a 1-truncated simplicial Lie algebra $\left\{G_{0}, G_{1}\right\}$ whose 1 -coskeleton we denote by $G^{1}$. The Lie algebra $M \ominus P$ acts on $M$ via the action of $P$ on $M$ and the homomorphism $d_{1}$. We can thus form the semi-direct product $M \ominus(M \ominus P)$ and construct homomorphisms

$$
\begin{array}{ll}
d_{0}: M \ominus(M \ominus P) \rightarrow M \ominus P, & \left(m, m^{\prime}, p\right) \mapsto\left(m^{\prime}, p\right), \\
d_{1}: M \ominus(M \ominus P) \rightarrow M \ominus P, & \left(m, m^{\prime}, p\right) \mapsto\left(m+m^{\prime}, p\right), \\
d_{2}: M \ominus(M \ominus P) \rightarrow M \ominus P, & \left(m, m^{\prime}, p\right) \mapsto\left(m, \partial m^{\prime}+p\right), \\
s_{0}: M \ominus P \rightarrow M \ominus(M \ominus P), & (m, p) \mapsto(0, m, p), \\
s_{1}: M \ominus P \rightarrow M \ominus(M \ominus P), & (m, p) \mapsto(m, 0, p) .
\end{array}
$$

Conditions (i) and (ii) of a crossed module ensure that these are homomorphisms (condition (ii) is needed for $\left.d_{2}\right)$. Let $G_{2}=M \ominus(M \ominus P)$. We then have a 2-truncated simplicial Lie algebra $\left\{G_{0}, G_{1}, G_{2}\right\}$ whose 2-coskeleton we denote by $\mathbf{G}^{2}$. There is a unique simplicial map $\mathbf{G}^{2} \rightarrow \mathbf{G}^{1}$ which in dimensions 0 and 1 is the identity. We let $\bar{G}^{2}$ denote the image of $G^{2}$ in $G^{1}$. It is readily checked that the Moore comple of $\mathbf{G}^{2}$ is trivial in dimension 2; it follows from Lemma 1 that $\overline{\mathbf{G}}^{2}$ is a simplicial Lie algebra whose Moore complex is of length 1 .

The above constructions yield the required equivalence.

We capture the structure of a Moore complex of length 2 in the following definition. A 2-crossed module is a pair of Lie homomorphisms

$$
L \stackrel{\delta}{\longrightarrow} M \stackrel{\partial}{\longrightarrow} P
$$

together with an action of $P$ on $M$, an action of $P$ on $L$, and a $\Lambda$-bilinear function $\{\}:, M \times M \rightarrow L$ such that axioms (1)-(8) hold for all $l, l^{\prime} \in L$, $m, m^{\prime}, m^{\prime \prime} \in M, p \in P$ : 
(1) $\partial \delta=0$

(2) $\delta\left({ }^{p} l\right)={ }^{p}(\delta l)$ and $\partial\left({ }^{p} m\right)=[p, m]$;

(3) $\delta\left\{m, m^{\prime}\right\}={ }^{(\partial m)} m^{\prime}-\left[m, m^{\prime}\right]$;

(4) $\left\{\delta l, \delta l^{\prime}\right\}=\left[l, l^{\prime}\right]$;

(5) $\{\delta l, m\}+\{m, \delta l\}={ }^{\partial m} l$;

(6) ${ }^{p}\left\{m, m^{\prime}\right\}=\left\{{ }^{p} m, m^{\prime}\right\}+\left\{m,{ }^{p} m^{\prime}\right\}$;

(7) $\left\{\left[m, m^{\prime}\right], m^{\prime \prime}\right\}={ }^{\partial m}\left\{m^{\prime}, m^{\prime \prime}\right\}+\left\{m,\left[m^{\prime}, m^{\prime \prime}\right]\right\}-{ }^{\partial m^{\prime}}\left\{m, m^{\prime \prime}\right\}$ $-\left\{m^{\prime},\left[m, m^{\prime \prime}\right]\right\}$;

(8) $\left\{m,\left[m^{\prime}, m^{\prime \prime}\right]\right\}={ }^{\partial m^{\prime}}\left\{m, m^{\prime \prime}\right\}-{ }^{\partial m^{\prime \prime}}\left\{m, m^{\prime}\right\}-\left\{m^{\prime}, \partial{ }^{\prime \prime} m^{\prime \prime}-\left[m, m^{\prime \prime}\right]\right\}$ $+\left\{m^{\prime \prime},{ }^{\partial m} m^{\prime}-\left[m, m^{\prime}\right]\right\}$.

A map of 2-crossed modules $(L \stackrel{\delta}{\rightarrow} M \stackrel{\partial}{\rightarrow} P) \rightarrow\left(L^{\prime} \stackrel{\delta^{\prime}}{\rightarrow} M^{\prime} \stackrel{\partial^{\prime}}{\rightarrow} P^{\prime}\right)$ is a triple of homomorphisms $f_{0}: P \rightarrow P^{\prime}, f_{i}: M \rightarrow M^{\prime}, f_{2}: L \rightarrow L^{\prime}$ such that $f_{0} \partial=\partial^{\prime} f_{1}, f_{1} \delta=\delta^{\prime} f_{2}$ and such that $f_{1}$ and $f_{2}$ are $f_{0}$-equivariant (that is, $f_{1}\left({ }^{p} m\right)={ }^{\left(f_{0} p\right)} f_{1}(m), f_{2}\left({ }^{p} l\right)={ }^{\left(f_{0} p\right)} f_{2}(l)$ for all $\left.l \in L, m \in M, p \in P\right)$.

THEOREM 3. The category of 2-crossed modules is equivalent to the category of simplicial Lie algebras with Moore complex of length 2.

Proof. Let $\mathbf{G}$ be a simplicial Lie algebra with Moore complex of length 2. We construct a 2-crossed module as follows: $P=G_{0}, M=\operatorname{ker}\left(d_{0}: G_{1} \rightarrow\right.$ $\left.G_{0}\right)$, and $L=\operatorname{ker}\left(d_{0}: G_{2} \rightarrow G_{1}\right) \cap \operatorname{ker}\left(d_{1}: G_{2} \rightarrow G_{1}\right)$. Then $p \in P$ acts on $m \in M$ by ${ }^{p} m=\left[s_{0} p, m\right]$, and on $l \in L$ by $\left[s_{0} s_{0} p, l\right]$. For $m, m^{\prime} \in M$ set $\left\{m, m^{\prime}\right\}=\left[s_{0} m-s_{1} m, s_{1} m^{\prime}\right]$. Let $\partial=d_{1}$ (restricted to $M$ ) and $\delta=d_{2}$ (restricted to $L$ ). It is routine to check that the axioms of a 2-crossed module hold.

Conversely suppose we start with a 2-crossed module $L \stackrel{\delta}{\longrightarrow} M \stackrel{\partial}{\longrightarrow} P$. Set $G_{0}=P$. Using the action of $P$ on $M$ we can form the semi-direct product $G_{1}=M \ominus P$. There are homomorphisms

$$
\begin{array}{ll}
d_{0}: M \ominus P \rightarrow P, & (m, p) \mapsto p, \\
d_{1}: M \ominus P \rightarrow P, & (m, p) \mapsto(\partial m)+p, \\
s_{0}: P \rightarrow M \ominus P, & p \mapsto(0, p) .
\end{array}
$$

There is an action of $m \in M$ on $l \in L$ given by

$$
m . l={ }^{\partial m} l-\{m, \delta l\} .
$$


Using this action we can form the semi-direct product $L \ominus M$. There is an action of $(m, p) \in M \ominus P$ on $\left(l, m^{\prime}\right) \in L \ominus M$ given by

$$
{ }^{(m, p)}\left(l, m^{\prime}\right)=\left({ }^{p} l+{ }^{\partial m} l+\left\{m, m^{\prime}\right\},{ }^{p} m^{\prime}+\left[m, m^{\prime}\right]\right) .
$$

Using this action we form the semi-direct product $G_{2}=(L \ominus M) \ominus(M \ominus P)$. (The $\Lambda$-bilinearity of $\{$,$\} together with axioms (6), (7) and (8) ensure that$ these last two actions are indeed Lie actions.) There are homomorphisms

$$
\begin{array}{ll}
d_{0}:(L \ominus M) \ominus(M \ominus P) \rightarrow(M \ominus P), & \left(l, m^{\prime}, m, p\right) \mapsto(m, p), \\
d_{1}:(L \ominus M) \ominus(M \ominus P) \rightarrow(M \ominus P), & \left(l, m^{\prime}, m, p\right) \mapsto\left(m^{\prime}+m, p\right), \\
d_{2}:(L \ominus M) \ominus(M \ominus P) \rightarrow(M \ominus P), & \left(l, m^{\prime}, m, p\right) \mapsto\left(m^{\prime}, \partial m+p\right), \\
s_{0}:(M \ominus P) \rightarrow(L \ominus M) \ominus(M \ominus P), & (m, p) \mapsto(0,0, m, p), \\
s_{1}:(M \ominus P) \rightarrow(L \ominus M) \ominus(M \ominus P), & (m, p) \mapsto(0, m, 0, p) .
\end{array}
$$

There is an action of $(l, m) \in L \ominus M$ on $l^{\prime} \in L$ given by

$$
{ }^{(l, m)} l^{\prime}=\left[l, l^{\prime}\right]+m \cdot l^{\prime},
$$

from which we can construct the semi-direct product $L \ominus(L \ominus M)$. There is an action of $(m, p) \in M \ominus P$ on $\left(l, l^{\prime}, m^{\prime}\right) \in L \ominus(L \ominus M)$ given by

$$
{ }^{(m, p)}\left(l, l^{\prime}, m^{\prime}\right)=\left({ }^{p} l+{ }^{\partial m} l,{ }^{p} l^{\prime}+{ }^{\partial m} l^{\prime},{ }^{p} m^{\prime}+\left[m, m^{\prime}\right]\right) \text {. }
$$

There is also an action of $\left(l^{\prime \prime}, m\right) \in L \ominus M$ on $\left(l, l^{\prime}, m^{\prime}\right) \in L \ominus(L \ominus M)$ given by

$$
\begin{aligned}
\left.l^{\prime \prime}, m\right)\left(l, l^{\prime}, m^{\prime}\right)= & \left(m . l+\left[l^{\prime \prime}, l\right]+\left\{m, \delta l^{\prime}\right\}+\left\{\delta l^{\prime \prime}, \delta l^{\prime}\right\}, m . l^{\prime}\right. \\
& \left.+\left[l^{\prime \prime}, l^{\prime}\right]+\left\{m, m^{\prime}\right\},\left[m, m^{\prime}\right]\right) .
\end{aligned}
$$

These last two actions combine to give an action of $(L \ominus M) \ominus(M \ominus P)$ on $L \ominus(L \ominus M)$, from which we construct the semi-direct product $G_{3}=$ $(L \ominus(L \ominus M)) \ominus((L \ominus M) \ominus(M \ominus P))$. There are homomorphisms

$$
\begin{aligned}
d_{0}: & (l \ominus(L \ominus M)) \ominus((L \ominus M) \ominus(M \ominus P)) \rightarrow((L \ominus M) \ominus(M \ominus P)) \\
& \left(l, l^{\prime}, m, l^{\prime \prime}, m^{\prime}, m^{\prime \prime}, p\right) \mapsto\left(l^{\prime \prime}, m^{\prime}, m^{\prime \prime}, p\right), \\
d_{1}: & (L \ominus(L \ominus M)) \ominus((L \ominus M) \ominus(M \ominus P)) \rightarrow((L \ominus M) \ominus(M \ominus P)) \\
& \left(l, l^{\prime}, m, l^{\prime \prime}, m^{\prime}, m^{\prime \prime}, p\right) \mapsto\left(l^{\prime}+l^{\prime \prime}, m+m^{\prime}, m^{\prime \prime}, p\right), \\
d_{2}: & (L \ominus(L \ominus M)) \ominus((L \ominus M) \ominus(M \ominus P)) \rightarrow((L \ominus M) \ominus(M \ominus P)) \\
& \left(l, l^{\prime}, m, l^{\prime \prime}, m^{\prime}, m^{\prime \prime}, p\right) \mapsto\left(l+l^{\prime}, m, m^{\prime}+m^{\prime}, p\right),
\end{aligned}
$$




$$
\begin{aligned}
d_{3}: & (L \ominus(L \ominus M)) \ominus((L \ominus M) \ominus(M \ominus P)) \rightarrow((L \ominus M) \ominus(M \ominus P)) \\
& \left(l, l^{\prime}, m, l^{\prime \prime}, m^{\prime}, m^{\prime \prime}, p\right) \mapsto\left(l, \delta l^{\prime}+m, \delta l^{\prime \prime}+m^{\prime}, \partial m^{\prime \prime}+p\right), \\
s_{0}: & (L \ominus M) \ominus(M \ominus P) \rightarrow(L \ominus(L \ominus M)) \ominus((L \ominus M) \ominus(M \ominus P)) \\
& \left(l, m, m^{\prime}, p\right) \mapsto\left(0,0,0,0, l, m, m^{\prime}, p\right), \\
s_{1}: & (L \ominus M) \ominus(M \ominus P) \rightarrow(L \ominus(L \ominus M)) \ominus((L \ominus M) \ominus(M \ominus P)) \\
& \left(l, m, m^{\prime}, p\right) \mapsto\left(0,0, l, m, 0,0, m^{\prime}, p\right), \\
s_{2}: & (L \ominus M) \ominus(M \ominus P) \otimes(L \ominus(L \ominus M)) \ominus((L \ominus M) \ominus(M \ominus P)) \\
& \left(l, m, m^{\prime}, p\right) \mapsto\left(0, l, 0, m, 0,0, m^{\prime}, p\right) .
\end{aligned}
$$

Axioms (1)-(5) ensure that these are indeed homomorphisms. Let $\mathbf{G}^{2}$ be the 2-coskeleton of the 2-truncated simplicial Lie algebra $\left\{G_{0}, G_{1}, G_{2}\right\}$; let $\mathbf{G}^{3}$ be the 3-coskeleton of the 3-truncated simplicial Lie algebra $\left\{G_{0}, G_{1}, G_{2}, G_{3}\right\}$. There is a unique simplicial map $\mathbf{G}^{3} \rightarrow \mathbf{G}^{2}$ which in dimensions 0,1 and 2 is the identity. We let $\overline{\mathbf{G}}^{3}$ denote the image of $\mathbf{G}^{3}$ in $\mathbf{G}^{2}$. It is readily checked that the Moore complex of $\mathbf{G}^{3}$ is trivial in dimension 3; it follows from Lemma 1 that $\overline{\mathbf{G}}^{3}$ is a simplicial Lie algebra whose Moore complex is of length 2.

The above constructions yield the required equivalence.

We now associate to the each simplicial Lie algebra $\mathbf{G}$ a simplicial inclusion $U^{k} \mathbf{G} \leadsto \mathbf{G}$ and quotient $\mathbf{G} \rightarrow V^{k} \mathbf{G}$ such that the following Proposition 4 holds. The inclusion and quotient are described carefully in the proof of Proposition 4, but in essence can be described in terms of Moore complexes as follows. Suppose that $\left(M_{n}, \partial_{n}\right)$ is the Moore complex of $\mathbf{G}$. Then $U^{k} \mathbf{G}$ will have Moore complex

$$
\cdots \rightarrow M_{k+3} \rightarrow M_{k+2} \rightarrow \operatorname{ker}\left(\partial_{k+1}\right) \rightarrow 0 \rightarrow 0 \cdots,
$$

and $V^{k} \mathbf{G}$ will have Moore complex

$$
\rightarrow 0 \rightarrow 0 \rightarrow \operatorname{im}\left(\partial_{k+1}\right) \nrightarrow M_{k} \rightarrow M_{k-1} \rightarrow \cdots .
$$

Proposition 4. For any simplicial Lie algebra $\mathbf{G}$ and integer $k \geq 0$ there is a functorial short exact sequence of simplicial Lie algebras

$$
0 \rightarrow U^{k} \mathbf{G} \stackrel{\iota}{\longrightarrow} \mathbf{G} \stackrel{\phi}{\longrightarrow} V^{k} \mathbf{G} \rightarrow 0
$$


such that:

(i) the Moore complex of $U^{k} \mathbf{G}$ is trivial in dimensions $0,1, \ldots, k$, and identical with the Moore complex of $\mathbf{G}$ in dimensions $\geq k+2$;

(ii) the map $l$ induces isomorphisms on homotopy groups $\pi_{n}\left(U^{k} \mathbf{G}\right) \cong$ $\pi_{n}(\mathbf{G})$ for $n \geq k+1$;

(iii) the Moore complex of $V^{k} \mathbf{G}$ is trivial in dimensions $\geq k+2$, and in dimensions $\leq k$ is identical with the Moore complex of $\mathbf{G}$.

Proof. First construct the $k$-coskeleton $\operatorname{cosk}^{k}\left(\operatorname{tr}^{k} \mathbf{G}\right)$ of $\mathbf{G}$. By Lemma 1 the Moore complex of $\operatorname{cosk}^{k}\left(\operatorname{tr}^{k} \mathbf{G}\right)$ is

$$
0 \rightarrow 0 \rightarrow K \stackrel{\partial_{k+1}}{\longrightarrow} M_{k} \stackrel{\partial_{k}}{\longrightarrow} M_{k-1} \rightarrow \cdots \stackrel{\partial_{1}}{\longrightarrow} M_{0} .
$$

Here $\partial_{k+1}$ is an inclusion, $K$ is the kernel of $\partial_{k}$, and in dimensions $\leq k$ this complex coincides with the Moore complex of $\mathbf{G}$. Since $K$ is an ideal in $G_{k}$, we can quotient $\operatorname{tr}^{k} \mathbf{G}$ by $K$ to obtain a $k$-truncated simplicial Lie algebra $\operatorname{tr}^{k} \mathbf{G} / K=\left\{G_{0}, \ldots, G_{k-1}, G_{k} / K\right\}$. We now construct the $k$-coskeleton $\operatorname{cosk}^{k}\left(\operatorname{tr}^{k} \mathbf{G} / K\right)$. There is a unique simplicial map $\mathbf{G} \rightarrow \operatorname{cosk}^{k}\left(\operatorname{tr}^{k} \mathbf{G} / K\right)$ which in dimensions less than $k$ is the identity, and in dimension $k$ is the quotient $G_{k} \rightarrow G_{k} / K$; we let $U^{k} \mathbf{G}$ denote the kernel of this map, and $V^{k} \mathbf{G}$ denote the image of $\mathbf{G}$ in $\operatorname{cosk}^{k}\left(\operatorname{tr}^{k} \mathbf{G} / K\right)$. We thus have a short exact sequence of simplicial Lie algebras

$$
0 \rightarrow U^{k} \mathbf{G} \rightarrow \mathbf{G} \rightarrow V^{k} \mathbf{G} \rightarrow 0
$$

which induces a long exact sequence of homotopy groups

$$
\cdots \rightarrow \pi_{n}\left(U^{k} \mathbf{G}\right) \rightarrow \pi_{n}(\mathbf{G}) \rightarrow \pi_{n}\left(V^{k} \mathbf{G}\right) \rightarrow \pi_{n-1}\left(U^{k} \mathbf{G}\right) \rightarrow \cdots
$$

The assertions of the proposition are easily checked.

Proposition 5. Let $\mathbf{G}$ be a simplicial Lie algebra such that $\pi_{n} \mathbf{G}=0$ for $n=0, \ldots, k$. Then there exists a weak homotopy equivalence $\mathbf{F} \simeq \mathbf{G}$ with F a free simplicial Lie algebra such that $F_{n}=0$ for $n=0, \ldots, k$.

Proof. It follows from Proposition 4(i) and the simplicial identities that the simplicial Lie algebra $U^{k} \mathbf{G}$ is trivial in dimensions $\leq k$. From axiom (M2) of a model category (see [16]) there is a weak equivalence $\mathbf{F} \simeq U^{k} \mathbf{G}$ 
with $\mathbf{F}$ a free simplicial Lie algebra. We can construct $\mathbf{F}$ so that it meets the requirements of the propositions.

\section{Free crossed and 2-crossed modules}

Suppose we are given a function $\xi: X \rightarrow P$ from a (possibly empty) set $X$ to a Lie algebra $P$. Then the free crossed module on $\xi$ is a crossed module $\partial: C(\xi) \rightarrow P$ satisfying:

i) $X$ is a subset of $C(\xi)$ and $\xi$ is the restriction of $\partial$;

ii) given any crossed module $\partial^{\prime}: M \rightarrow P$ and function $\phi: X \rightarrow M$ such that $\partial^{\prime} \phi(x)=\partial(x)$ for all $x \in X$, the function $\phi$ extends uniquely to a map of crossed modules $\phi: C(\xi) \rightarrow M$ over the identity on $P$.

The free crossed module exists for any $\xi$ (we recall its construction below from [10]) and is clearly uniquely determined up to isomorphism by $\xi$.

If $P$ is a free Lie algebra, we say that the crossed module $\partial: C(\xi) \rightarrow P$ is totally free.

It will be helpful to have the notion of a precrossed module: this is just a Lie homomorphism $\partial: M \rightarrow P$ with an action of $P$ on $M$ satisfying $\pi\left({ }^{p} m\right)=$ $[p, \partial m]$ for $m \in M, p \in P$. On replacing "crossed" by "precrossed" in the above definition of a (totally) free crossed module we obtain the appropriate definition of a (totally) free precrossed module.

We construct the free crossed module on $\xi: X \rightarrow P$ as follows. Let $\Lambda_{X}$ be the free $\Lambda$-module on $X$, and let $\partial: \Lambda_{X} \rightarrow P$ be the unique module homomorphism satisfying $\partial(x)=\xi(x)$. Let $P^{e}$ be the universal enveloping algebra of $P$, and form the tensor product of $\Lambda$-modules $Q=P^{e} \otimes \Lambda_{X}$. There is a canonical action of $P$ on $Q$ which, if we think of $Q$ as an abelian Lie algebra, is an action of Lie algebras. There is a unique $P$-equivariant homomorphism $\partial: Q \rightarrow P$ such that $\partial(1 \otimes v)=\partial v$ for all $v \in \Lambda_{X}$. Let $\mathscr{A}$ be the free Lie algebra on the $\Lambda$-module $Q$, and $\partial: \mathscr{A} \rightarrow P$ the unique Lie homomorphism extending $\partial: Q \rightarrow P$. The action of $P$ on $Q$ extends uniquely to an action of $P$ on $\mathscr{A}$, and the Lie homomorphism $\partial: \mathscr{A} \rightarrow P$ is a precrossed module: it is the free precrossed module on $\xi$. Let $\mathscr{I}$ be the ideal of $\mathscr{A}$ generated by the Peiffer elements

$$
{ }^{\partial a} b-[a, b]
$$

for $a, b \in \mathscr{A}$. Put $C(\xi)=\mathscr{A} / \mathscr{I}$. The induced homomorphism $\partial: C(\xi) \rightarrow$ $P$ inherits the structure of the free crossed module on $\xi$.

Suppose now we are given a precrossed module $\partial: M \rightarrow P$ and a function $\theta: Y \rightarrow \operatorname{ker} \partial$ from a (possibly empty) set $Y$ to the kernel of $\partial$. The free 2-crossed module on $\theta$ is a 2-crossed module $C(\theta) \stackrel{\delta}{\longrightarrow} M \stackrel{\partial}{\longrightarrow} P$ satisfying: 
i) $Y$ is a subset of $C(\vartheta)$ and $\vartheta$ is the restriction of $\delta$;

ii) given any 2-crossed module $L \stackrel{\delta^{\prime}}{\rightarrow} M \rightarrow P$ and function $\phi: Y \rightarrow L$ such that $\delta^{\prime} \phi(y)=\delta(y)$ for all $y \in Y$, the function $\phi$ extends uniquely to a map of 2-crossed modules $\phi: C(\vartheta) \rightarrow L$ over the identity on $M$ and $P$.

The free 2-crossed module is uniquely determined up to isomorphism by $\vartheta$. If $\partial: M \rightarrow P$ is a totally free precrossed module, we say that the 2 -crossed module $C(\vartheta) \stackrel{\delta}{\rightarrow} M \stackrel{\partial}{\rightarrow} P$ is totally free.

We shall give an explicit description of the construction of a free 2-crossed module in one special case only, namely when $\partial: M \rightarrow P$ is an arbitrary precrossed module, $Y=\varnothing$ is the empty set, and $\vartheta: \varnothing \rightarrow M$ the unique "function" from $\varnothing$ to $M$. For this we need to define the non-abelian tensor product $M \otimes_{P} M$; we take this to be the Lie algebra generated by the symbols $x \otimes y$ with $x, y \in M$, subject to the following relations for all $\lambda \in \Lambda$, $x, x^{\prime}, y, y^{\prime} \in M$ and $p \in P$ :

$$
\begin{gathered}
\lambda(x \otimes y)=(\lambda x \otimes y)=(x \otimes \lambda y), \\
\left(x+x^{\prime}\right) \otimes y=x \otimes y+x^{\prime} \otimes y, \\
x \otimes\left(y+y^{\prime}\right)=x \otimes y+x \otimes y^{\prime}, \\
{\left[x, x^{\prime}\right] \otimes y={ }^{\partial x}\left(x^{\prime} \otimes y\right)+x \otimes\left[x^{\prime}, y\right]-{ }^{\partial x^{\prime}}(x \otimes y)-x^{\prime} \otimes[x, y],} \\
x \otimes\left[y, y^{\prime}\right]={ }^{\partial y}\left(x \otimes y^{\prime}\right)-{ }^{\partial y^{\prime}}(x \otimes y) \\
-y \otimes\left({ }^{x x} y^{\prime}-\left[x, y^{\prime}\right]\right)+y^{\prime} \otimes\left({ }^{\partial x} y-[x, y]\right),
\end{gathered}
$$

where by definition

$$
{ }^{p}(x \otimes y)=\left({ }^{p} x \otimes y\right)+\left(x \otimes{ }^{p} x\right) .
$$

(As a consequence of identities (9), (10), (11) and (14) we can view $M \otimes_{P} M$ as a quotient of the standard $\Lambda$-module tensor product $M \otimes_{\Lambda} M$ in which $M$ is considered as a $\Lambda$-module.)

Proposition 6. (i) There is an action of $P$ on $M \otimes_{P} M$ defined by

$$
{ }^{p}(x \otimes y)=\left({ }^{p} x \otimes y\right)+\left(x \otimes{ }^{p} y\right)
$$

for $p \in P, x, y \in M$. 
(ii) There is a P-equivariant homomorphism $\delta: M \otimes_{P} M \rightarrow M$ defined by

$$
\delta(x \otimes y)={ }^{\partial x} y-[x, y]
$$

(iii) The pair of homomorphisms

$$
M \otimes_{P} M \stackrel{\delta}{\longrightarrow} M \stackrel{\partial}{\longrightarrow} P
$$

together with the action given in (i), the action of $P$ on $M$, and the function $\{\}:, M \times M \rightarrow M \otimes_{P} M,(x, y) \mapsto x \otimes y$ is the free 2-crossed module on the "function" $\varnothing \rightarrow M$.

Proof. (i) Let $F$ be the free Lie algebra generated by the symbols $x \otimes y$ with $x, y \in M$, and let $I$ be the ideal of $F$ generated by the relations (9)-(15). There is clearly a unique action of $P$ on $F$ defined on generators by

$$
{ }^{p}(x \otimes y)=\left({ }^{p} x \otimes y\right)+\left(x \otimes \otimes^{p} y\right) .
$$

It is routine to check that $I$ is invariant under this action. Hence $P$ acts on $M \otimes_{P} M$ as required.

(ii) It is also routine to check that the homomorphism $\delta: F \rightarrow M$ defined on generators by $\delta(x \otimes y)={ }^{\partial x} y-[x, y]$ sends $I$ to the 0 , and is $P$ equivariant. Hence the required homomorphism $\delta: M \otimes_{P} M \rightarrow M$ exists.

(iii) This is clear.

In [10] a tensor product $M \otimes M$ was defined for an arbitrary Lie algebra $M$. It is generated by symbols $x \otimes y$ for $x, y \in M$ subject to the above relations (9), (10), (11) and (14) and the following relations for $x, x^{\prime}, y, y^{\prime} \in M$ :

$$
\begin{aligned}
& {\left[x, x^{\prime}\right] \otimes y=x \otimes\left[x^{\prime}, y\right]-x^{\prime} \otimes[x, y],} \\
& x \otimes\left[y, y^{\prime}\right]=\left[y^{\prime}, x\right] \otimes y-[y, x] \otimes y^{\prime} .
\end{aligned}
$$

It is routine exercise to show that $M \otimes M$ is precisely the tensor product $M \otimes_{P} M$ defined above with $P=0$.

We now recall from [17] a generalisation of a construction of Whitehead. The universal quadratic functor $\Gamma$ is defined for any $\Lambda$-module $\pi$ to be the $\Lambda$-module $\Gamma(\pi)$ generated by the symbols $\gamma(x)$ for $x \in \pi$, subject to the relations

(19) $\gamma(x+y+z)+\gamma(x)+\gamma(y)+\gamma(z)=\gamma(x+y)+\gamma(x+z)+\gamma(y+z)$,

$$
\gamma(\lambda x+y)+\lambda \gamma(x)+\lambda \gamma(y)=\lambda \gamma(x+y)+\gamma(\lambda x)+\gamma(y) \text {, }
$$

for all $\lambda \in \lambda, x, y, z \in \pi$.

The following result is a Lie algebraic version of Theorem (IV) 1.8 in [1]. 
Theorem 7. Suppose that the ground ring $\Lambda$ is a field. Let $\partial: M \rightarrow P$ be a totally free precrossed module. Then there is an isomorphism

$$
\Gamma\left(\pi_{1}\right) \cong \operatorname{ker}\left(M \otimes_{P} M \rightarrow M\right)
$$

where $\pi_{1}=\operatorname{ker}\left(M / \delta\left(M \otimes_{p} M\right) \rightarrow P\right)$.

PROof. The precrossed module gives rise to a 1-truncated free simplicial Lie algebra $M \stackrel{\leftarrow}{\Rightarrow} P$ whose 1 -skeleton we denote by $F$. The beginning of the Moore complex of $\mathbf{F}$ is $M_{3} \rightarrow M_{2} \rightarrow M \rightarrow P$. Universal properties imply that $M \otimes_{p} M \cong M_{2} / \operatorname{im}\left(M_{3}\right)$. Hence $\pi_{2}(\mathrm{~F}) \cong \operatorname{ker}\left(M \otimes_{P} M \rightarrow M\right)$. Theorems 10 and 11 of the next section yield the exact sequence

$$
0=H_{3}(\mathbf{F}) \rightarrow \Gamma\left(\pi_{1}(\mathbf{F})\right) \rightarrow \pi_{2}(\mathbf{F}) \rightarrow H_{2}(\mathbf{F})=0 .
$$

The theorem follows.

\section{Lie algebraic analogues of classical theorems}

The following result is a Lie algebraic version of the Hurewicz Theorem. It could have been deduced from Sublemma 8.3 in [6], but instead we give an alternative and simpler proof using Theorem 3.

THEOREM 8. For any simplicial Lie algebra $\mathbf{G}$ there is an isomorphism

$$
H_{0}(\mathbf{G}) \cong \pi_{0}(\mathbf{G})^{\mathrm{ab}} \text {. }
$$

If $\pi_{i} \mathbf{G}=0$ for $0 \leq i \leq k$, then there is an isomorphism

$$
\pi_{k+1} \mathbf{G} \cong H_{k+1} \mathbf{G}
$$

and a surjection

$$
\pi_{k+2} \mathbf{G} \rightarrow H_{k+2} \mathbf{G} .
$$

Proof. The first isomorphism is clear. Suppose $\pi_{i} \mathbf{G}=0$ for $0 \leq i \leq k$. By Proposition 5 we can choose a free simplicial Lie algebra $F$ which is weakly homotopic to $\mathbf{G}$ and which is trivial in dimensions $\leq k$. The Moore complex of $F$ is of the form

$$
\rightarrow M_{k+3} \rightarrow M_{k+2} \rightarrow M_{k+1} \rightarrow 0 \rightarrow 0 \rightarrow \cdots \text {. }
$$

Now

$$
\cdots \rightarrow M_{k+3} \rightarrow M_{k+2} \rightarrow M_{k+1}
$$

is the beginning of the Moore complex of the simplicial Lie algebra $\operatorname{Dec}_{k} \mathbf{F}$ obtained from $\mathbf{F}$ by forgetting: the first $k+1$ dimensions of $\mathbf{F}$; the first 
$k+2$ boundary maps; and the first $k+1$ degeneracy maps (cf. [8]). We thus have the bracket $\{\}:, M_{k+1} \times M_{k+1} \rightarrow M_{k+2}$. In dimension $k+$ 2 the Moore complex of [F, F] contains $\left\{M_{k+1}, M_{k+1}\right\}$ and hence, using essentially identity (3) with $\partial m=0$, we see that $\pi_{k+1}[\mathbf{F}, \mathbf{F}]=0$. The short exact sequence of simplicial Lie algebras

$$
[\mathbf{F}, \mathbf{F}] \nrightarrow \mathbf{F} \rightarrow \mathbf{F}^{\mathbf{a b}}
$$

gives rise to the long exact sequence of homotopy groups

$$
\rightarrow \pi_{k+2}[\mathbf{F}, \mathbf{F}] \rightarrow \pi_{k+2} \mathbf{F} \rightarrow \pi_{k+2} \mathbf{F}^{\mathrm{ab}} \rightarrow 0 \rightarrow \pi_{k+1} \mathbf{F} \rightarrow \pi_{k+1} \mathbf{F}^{\mathrm{ab}} \rightarrow 0
$$

from which the isomorphism and surjection of the theorem follows.

It would be nice to have a proof of the following Lie algebraic version of the Whitehead Theorem which, unlike the proof described in [6], does not rely on the connectivity Sublemma 8.3 of [6]. We outline the proof described in [6]. In stating the result we say that a simplicial Lie algebra is connected to mean that the 0th homotopy group $\pi_{0}$ is trivial.

THEOREM 9. Suppose that the ground ring $\Lambda$ is a field. Let $f: \mathbf{E} \rightarrow \mathbf{G}$ be a map of connected simplicial Lie algebraic which induces isomorphisms $H_{n}(\mathbf{E}) \cong H_{n}(\mathbf{G})$ on homology for $n \geq 0$. Then $f$ is a weak homotopy equivalence.

Outline Proof. We need some notation. For any Lie algebra $G$ we let $\gamma_{1} G=G$ and define inductively $\gamma_{i} G=\left[\gamma_{i-1} G, G\right]$. For any simplicial Lie algebra $\mathbf{G}$ we let $\gamma_{i} \mathbf{G}$ be the simplicial Lie algebra obtained by applying $\gamma_{i}$ dimensionwise. We let $\bar{H}_{n}(G)$ denote the $n$th Eilenberg-MacLane homology of a Lie algebra $G$ with coefficients in $\Lambda$. (The definition of $\bar{H}_{n}(G)$ is recalled in Section 6; in that section we revert to standard notation and write $H_{n}(G)$ in place of $\bar{H}_{n}(G)$.)

To prove the theorem we can assume that $\mathbf{E}$ and $\mathbf{G}$ are free. We are given that the induced map $f^{2}: \mathbf{E}^{\mathrm{ab}} \rightarrow \mathbf{G}^{\mathrm{ab}}$ is a weak equivalence of simplicial vector spaces; any such weak equivalence is actually a homotopy equivalent since the category of simplicial vector spaces is a model category [16]. We shall show by induction that the induced simplicial map $f^{n}: \mathbf{E} / \gamma_{n} \mathbf{E} \rightarrow \mathbf{G} / \gamma_{n} \mathbf{G}$ is a homotopy equivalence for all $n$. As the inductive hypothesis, suppose that $f^{n-1}$ is a homotopy equivalence. Applying $\bar{H}_{2}(-)$ dimensionwise to 
$\mathbf{E} / \gamma_{n} \mathbf{E}$ and $\mathbf{G} / \gamma_{n} \mathbf{G}$, we get the following diagram of simplicial vector spaces:

$$
\begin{array}{cc}
\bar{H}_{2}\left(\mathbf{E} / \gamma_{n-1} \mathbf{E}\right) \stackrel{\cong}{\longrightarrow} \gamma_{n-1} \mathbf{E} / \gamma_{n} \mathbf{E} \\
\lfloor\cong \\
\bar{H}_{2}\left(\mathbf{G} / \gamma_{n-1} \mathbf{G}\right) \stackrel{ }{\longrightarrow} \gamma_{n-1} \mathbf{G} / \gamma_{n} \mathbf{G}
\end{array}
$$

The two indicated isomorphisms follow from Hopf's formula for $\bar{H}_{2}(-)$ [13]. The indicated homotopy equivalence follows from the fact that $\bar{H}_{2}$ is a functor and thus preserves homotopy equivalences. We thus have a homotopy equivalence $\gamma_{n-1} \mathbf{E} / \gamma_{n} \mathbf{E} \simeq \gamma_{n-1} \mathbf{G} / \gamma_{n} \mathbf{G}$. The long exact homotopy sequences arising from the diagram

$$
\begin{array}{r}
\gamma_{n-1} \mathbf{E} / \gamma_{n} \mathbf{E} \multimap \mathbf{E} / \gamma_{n} \mathbf{E} \rightarrow \mathbf{E} / \gamma_{n-1} \mathbf{E} \\
\downarrow \cong \quad \downarrow \\
\gamma_{n-1} \mathbf{G} / \gamma_{n} \mathbf{G} \multimap \mathbf{E} / \gamma_{n} \mathbf{G} \rightarrow \mathbf{E} / \gamma_{n-1} \mathbf{G}
\end{array}
$$

yield a commutative diagram

$$
\begin{aligned}
& \rightarrow \pi_{i}\left(\gamma_{n-1} \mathbf{E} / \gamma_{n} \mathbf{E}\right) \rightarrow \pi_{i}\left(\mathbf{E} / \gamma_{n} \mathbf{E}\right) \rightarrow \pi_{i}\left(\mathbf{E} / \gamma_{n-1} \mathbf{E}\right) \rightarrow \pi_{i-1}\left(\gamma_{n-1} \mathbf{E} / \gamma_{n} \mathbf{E}\right) \rightarrow
\end{aligned}
$$

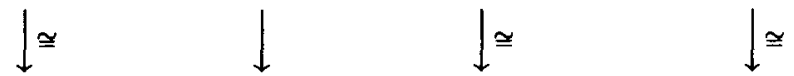

$$
\begin{aligned}
& \rightarrow \pi_{i}\left(\gamma_{n-1} \mathbf{G} / \gamma_{n} \mathbf{G}\right) \rightarrow \pi_{i}\left(\mathbf{G} / \gamma_{n} \mathbf{G}\right) \rightarrow \pi_{i}\left(\mathbf{G} / \gamma_{n-1} \mathbf{G}\right) \rightarrow \pi_{i-1}\left(\gamma_{n-1} \mathbf{G} / \gamma_{n} \mathbf{G}\right) \rightarrow
\end{aligned}
$$

in which the rows are exact; the indicated isomorphisms arise from the homotopy equivalences. We can conclude that there are isomorphisms $\pi_{i}\left(\mathbf{E} / \gamma_{n} \mathbf{E}\right)$ $\cong \pi_{i}\left(\mathbf{G} / \gamma_{n} \mathbf{G}\right)$ for all $i \geq 0$. However, any weak homotopy equivalence between two simplicial free nilpotent Lie algebras of class $n$ is a homotopy equivalence, since simplicial nilpotent Lie algebras form a model category [16]. It follows by induction that $f^{n}: \mathbf{E} / \gamma_{n} \mathbf{E} \rightarrow \mathbf{G} / \gamma_{n}$ is a homotopy equivalence for all $n$. Sublemma 8.3 in [6], which we do not prove, tells us that $\pi_{q}\left(\gamma_{n} \mathbf{E}\right)=\pi_{q}\left(\gamma_{n} \mathbf{G}\right)=0$ for $q \leq \log _{2} n$. What is important here is that as $n$ tends to infinity, so does the connectivity of $\gamma_{n} \mathbf{E}$ and $\gamma_{n} \mathbf{G}$. Thus for any given $m$ we can choose an $n$ such that the quotients $\mathbf{E} \rightarrow \gamma_{n} \mathbf{E}, \mathbf{G} \rightarrow \gamma_{n} \mathbf{G}$ induce isomorphisms on homotopy groups $\pi_{q}$ for $q \leq m$. It follows that $f: \mathbf{E} \rightarrow \mathbf{G}$ is a weak homotopy equivalence.

The next theorem is analogous to the topological fact that the homology of a $k$-dimensional space, and also the homology of its universal cover, is trivial in dimensions greater than $k$. In the hypothesis of the theorem the condition that $\Lambda$ be a field is not essential, but is included to simplify the proof. 
The universal cover $\widetilde{\mathbf{G}}$ of a simplicial Lie algebra $\mathbf{G}$ is obtained as follows. Let $\mathbf{B}(\pi)$ be the simplicial Lie algebra which in each dimension is equal to $\pi=\pi_{0}(\mathbf{G})$, and in which all boundary and degeneracy maps are the identity. Then $\widetilde{\mathbf{G}}$ is the kernel of the canonical surjection $\mathbf{G} \rightarrow \mathbf{B}(\pi)$. It follows that $\pi_{n}(\widetilde{\mathbf{G}}) \cong \pi_{n}(\mathbf{G})$ for $n \geq 1$ and $\pi_{0}(\widetilde{\mathbf{G}})=0$.

THEOREM 10. Let $\Lambda$ be a field. The homology $H_{n}\left(\mathrm{sk}^{k}\left(\mathrm{tr}^{k} \mathrm{G}\right)\right)$ of the $k$ skeleton of a free simplicial Lie algebra $\mathbf{F}$ is trivial for $n \geq k+1$. The homology $H_{n}(\widetilde{\mathbf{G}})$ of the universal cover $\widetilde{\mathbf{G}}$ of $\mathbf{s k}^{k}\left(\mathrm{tr}^{k} \mathbf{F}\right)$ is also trivial for $n \geq k+1$.

Proof. Note that $\mathbf{s k}^{k}\left(\operatorname{tr}^{k} \mathbf{F}\right)$ is a free simplicial Lie algebra. According to the Dold-Kan Theorem [7] the Moore complex functor induces an equivalence between the category of simplicial $\Lambda$-modules and the category chain complexes of $\Lambda$-modules. Under this equivalence the simplicial $\Lambda$-module $\left(\mathbf{s k}^{k}\left(\operatorname{tr}^{k} \mathbf{F}\right)\right)^{\mathrm{ab}}$ corresponds to a chain complex which is trivial in dimensions higher than $k$. Hence the homology of $\operatorname{sk}^{k}\left(\operatorname{tr}^{k} \mathbf{F}\right)$ is trivial in dimension higher than $k$.

If $\Lambda$ is a field then any subalgebra of a free Lie algebra is free [18]. Hence $\widetilde{\mathbf{G}}$ is a free simplicial Lie algebra. Under the Dold-Kan equivalence $\widetilde{\mathbf{G}}^{\text {ab }}$ corresponds to a chain complex of $\pi_{0}(\mathbf{F})$-modules which is trivial in dimensions higher than $k$.

The next theorem is a Lie algebraic version of Whitehead's Certain Exact Sequence [19].

THEOREM 11. Suppose that the ground ring $\Lambda$ is a field. Let $\mathbf{G}$ be a simplicial Lie algebra with $\pi_{0}(\mathbf{G})=0$. Then there is an exact sequence

$$
\pi_{3}(\mathbf{G}) \rightarrow H_{3}(\mathbf{G}) \rightarrow \Gamma\left(\pi_{1}(\mathbf{G})\right) \rightarrow \pi_{2}(\mathbf{G}) \rightarrow H_{2}(\mathbf{G}) \rightarrow 0 .
$$

Proof. From the proof of Theorem 8 we have an exact sequence

$$
\pi_{3}(\mathbf{F}) \rightarrow H_{3}(\mathbf{F}) \rightarrow \pi_{2}([\mathbf{F}, \mathbf{F}]) \rightarrow \pi_{2}(\mathbf{F}) \rightarrow H_{2}(\mathbf{F}) \rightarrow 0
$$

where $\mathbf{F}$ is a free simplicial Lie algebra that is weakly homotopic to $\mathbf{G}$ and trivial in dimension 0 . Thus we have only to prove that $\pi_{2}([\mathbf{F}, \mathbf{F}])$ is isomorphic to $\Gamma\left(\pi_{1}(\mathbf{F})\right)$. Suppose that the beginning of the Moore complex of $F$ is $\rightarrow M_{3} \rightarrow M_{2} \rightarrow M \rightarrow 0$. Let $L=M_{2} / \operatorname{im}\left(M_{3}\right)$. Then $L \rightarrow M \rightarrow 0$ is a 2crossed module. It is readily checked that the 2-crossed module obtained in a similar fashion from the Moore complex of $[\mathbf{F}, \mathbf{F}]$ is $[L, L] \rightarrow[M, M] \rightarrow 0$. 
We have a commutative diagram

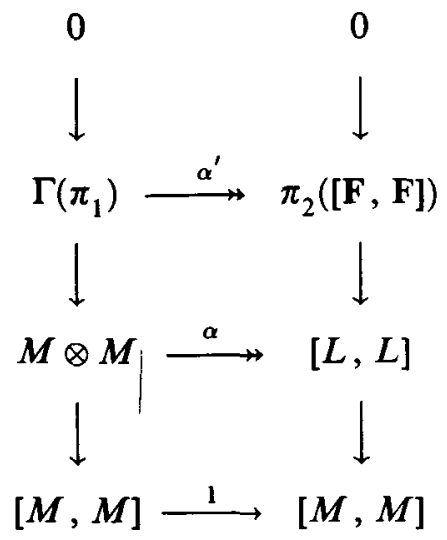

in which the columns are exact, and $\pi_{1}=M^{\mathrm{ab}}$. Here $M \otimes M=M \otimes_{P} M$ with $P=0$. The exactness of the left-hand column follows from [10, Theorem 18]. The surjectivity of $\alpha$ is due to identity (4), and implies the surjectivity of $\alpha^{\prime}$. Let $\pi_{1} / L$ denote $M / \delta L=\pi_{1}(F)$. Then, by identity (4), $\alpha^{\prime}$ induces a map $\alpha^{\prime \prime}: \Gamma\left(\pi_{1} / L\right) \rightarrow \pi_{2}([\mathbf{F}, \mathbf{F}])$. Since $L \rightarrow M \rightarrow 0$ is a free 2 -crossed module we can construct a map of 2-crossed modules

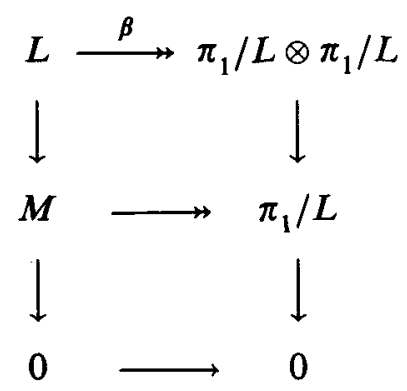

Since $\pi_{1} / L$ is a vector space, it follows again from [10, Theorem 18] that $\Gamma\left(\pi_{1} / L\right)=\operatorname{ker}\left(\pi_{1} / L \otimes \pi_{1} / L \rightarrow \pi_{1} / L\right)$. The restriction of $\beta$ to $[L, L]$ induces a homomorphism $\beta^{\prime}: \pi_{2}([\mathbf{F}, \mathbf{F}]) \rightarrow \Gamma\left(\pi_{1} / L\right)$. The homomorphisms $\alpha^{\prime \prime}$ and $\beta^{\prime}$ are mutually inverse. Hence $\pi_{2}([F, F])$ is isomorphic to $\Gamma\left(\pi_{1}(F)\right)$.

The following theorem is a Lie algebraic version of the Mayer-Vietoris homology sequence. The statement of the theorem involves the notion of a free simplicial map, which was recalled in Section 1. 
THEOREM 12. Let

$$
\begin{array}{r}
\mathbf{G} \stackrel{i^{\prime}}{\longrightarrow} \mathbf{G}^{\prime} \\
i^{\prime \prime} \downarrow \text { pushout } \downarrow \\
\mathbf{G}^{\prime \prime} \longrightarrow \mathbf{K}
\end{array}
$$

be a pushout of simplicial Lie algebras in which $i^{\prime}$ and $i^{\prime \prime}$ are free maps, and $\mathbf{G}$ a free simplicial Lie algebra. Then there is a long exact sequence

$$
\rightarrow H_{n}(\mathbf{G}) \rightarrow H_{n}\left(\mathbf{G}^{\prime}\right) \oplus H_{n}\left(\mathbf{G}^{\prime \prime}\right) \rightarrow H_{n}(\mathbf{K}) \rightarrow H_{n-1}(\mathbf{G}) \rightarrow
$$

Proof. Since $\mathbf{G}$ is free and $i^{\prime}, i^{\prime \prime}$ are free maps it follows that $\mathbf{K}$ is free. In each dimension $n$ we have a pushout of free Lie algebras

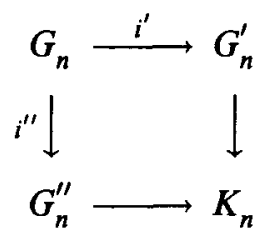

The Mayer-Vietoris sequence in the homology of Lie algebras [13], and the fact that the second homology of a free Lie algebra is trivial, imply a short exact sequence $\left(G_{n}\right)^{\mathrm{ab}} \mapsto\left(G_{n}^{\prime}\right)^{\mathrm{ab}} \oplus\left(G_{n}^{\prime \prime}\right)^{\mathrm{ab}} \rightarrow\left(K_{n}\right)^{\mathrm{ab}}$. We thus have a short exact sequence of simplicial modules $\mathbf{G}^{\mathrm{ab}} \mapsto \mathbf{G}^{\mathrm{ab}} \oplus \mathbf{G}^{\prime \prime a b} \rightarrow \mathbf{K}^{\mathrm{ab}}$ the long exact homotopy sequence of which is the required sequence.

The following theorem is a Lie algebraic version of the exact sequence given in [3, Chapter VII, exercise 6] (see also [11, Theorem 10]). The simplicial Lie algebra $\mathbf{B}(\pi)$ used in the theorem is described above.

TheOREM 13. For any simplicial Lie algebra $\mathbf{G}$ there is an exact homology sequence

$$
H_{2}(\mathbf{G}) \rightarrow H_{2}(\mathbf{B}(\pi)) \rightarrow\left(\pi_{1}(\mathbf{G})\right)_{\pi} \rightarrow H_{1}(\mathbf{G}) \rightarrow H_{1}(\mathbf{B}(\pi)) \rightarrow 0
$$

where $\pi=\pi_{0}(\mathbf{G})$ and $\left(\pi_{1}(\mathbf{G})\right)_{\pi}$ is obtained from $\pi_{1}(\mathbf{G})$ by killing the action of $\pi$.

Proof. There is a canonical map $\mathbf{G} \rightarrow \mathbf{B}(\pi)$. Since we are essentially only interested in the weak homotopy type of simplicial Lie algebras we can assume (thanks to the axioms of a model category) that both $\mathbf{G}$ and $\mathbf{B}(\pi)$ are free simplicial Lie algebras, and that $\mathbf{G} \rightarrow \mathbf{B}(\pi)$ is a free map. Also we can assume that $\mathbf{G}$ and $\mathbf{B}(\pi)$ are identical in dimensions 0 and 1. Since $\mathbf{G} \rightarrow \mathbf{B}(\pi)$ is a free map it follows that the induced simplicial map $\mathbf{G}^{\mathrm{ab}} \mapsto$ 
$(\pi)^{a b}$ is injective. Let $\mathbf{C}$ be the cokernel of $\mathbf{G}^{\mathrm{ab}} \rightarrow \mathbf{B}(\pi)^{\mathrm{ab}}$. Part of the long exact homotopy sequence arising from $\mathbf{G} \leadsto \mathbf{B}(\pi)^{\mathrm{ab}} \rightarrow \mathbf{B}$ is thus

$$
H_{2}(\mathbf{G}) \rightarrow H_{2}(\mathbf{B}(\pi)) \rightarrow \pi_{2}(\mathbf{C}) \rightarrow H_{1}(\mathbf{G}) \rightarrow H_{1}(\mathbf{B}(\pi)) \rightarrow \pi_{1}(\mathbf{C})=0 .
$$

It remains to identify $\pi_{1}(\mathbf{C})$. Considering the map $\mathbf{G} \mapsto \mathbf{B}(\pi)$ as a map of simplicial modules, we can form its cokernel $\overline{\mathbf{C}}$. The long exact homotopy sequence arising from $\mathbf{G} \rightarrow \mathbf{B}(\pi) \rightarrow \overline{\mathbf{C}}$ implies that $\pi_{2}(\overline{\mathbf{C}}) \cong \pi_{1}(\mathbf{G})$. It is thus readily seen that $\pi_{2}\left(\mathbf{C} \cong\left(\pi_{1}(\mathbf{G})\right)_{\pi}\right.$.

\section{Coproducts of crossed modules}

There is a canonical functor

$$
\chi:(\text { simplicial Lie algebras }) \rightarrow \text { (crossed modules })
$$

which sends a simplicial Lie algebra $\mathbf{G}$ with Moore complex $\cdots \rightarrow M_{2} \stackrel{\delta}{\rightarrow}$ $M_{1} \stackrel{\partial}{\rightarrow} M_{0}$ to the crossed module $\chi(\mathbf{G})$ consisting of the induced map $\partial: M_{1} / \delta\left(M_{2}\right) \rightarrow M_{0}$ and standard action (cf. Proposition 4). The crossed module $\chi(\mathbf{G})$ is equivalent to the simplicial Lie algebra obtained from $\mathbf{G}$ by factoring out the terms of the Moore complex of dimension $\geq 2$. It follows that the functor $\chi$ preserves coproducts. In order to utilise this property of $\chi$ we need a description of coproducts in the category of crossed modules.

Let $\partial_{L}: L \rightarrow P, \partial_{M}: M \rightarrow P, \partial_{N}: N \rightarrow P$ be crossed modules and suppose given two maps $\left(\partial_{L}: L \rightarrow P\right) \rightarrow\left(\partial_{M}: M \rightarrow P\right)$ and $\left(\partial_{L}: L \rightarrow P\right) \rightarrow$ $\left(\partial_{M}: M \rightarrow P\right)$ both over the identity on $P$. There is a pushout diagram of the form

$$
\begin{array}{cc}
(\partial: L \rightarrow P) & \longrightarrow \\
\downarrow & \left(\partial_{N}: N \rightarrow P\right) \\
\left(\partial_{N}: L \rightarrow P\right) & \text { pushout } \downarrow
\end{array}
$$

We shall give an explicit construction for the crossed module $\partial: M^{L} N \rightarrow P$.

The Lie algebras $M, N$ act on each other via the action of $P$. Ignoring for the moment the action of $M$ on $N$, we can form the semi-direct $M \ominus N$ and homomorphism j $\bar{\partial}: M \ominus N \rightarrow P,(m, n) \mapsto \partial_{M}(m)+\partial_{N}(n)$. There is an action of $p \in P$ on $(m, n) \in M \ominus N$ given by ${ }^{p}(m, n)=\left({ }^{p} m,{ }^{p} n\right)$. With this action the homomorphism $\bar{\partial}$ is a precrossed module. Let $I$ be the $P$-invariant ideal of $M \ominus N$ generated by the Peiffer elements

$$
\left[(m, n),\left(n^{\prime}, n^{\prime}\right)\right]-^{\bar{\partial}(m, n)}\left(m^{\prime}, n^{\prime}\right)
$$


for $m, n^{\prime} \in M, n, n^{\prime} \in N$. It is readily checked that $I$ is generated, as an abelian group, by the elements

$$
\left({ }^{\partial n} m,{ }^{\partial m} n\right)
$$

for $\in M, n \in N$.

There is a homomorphism $l: A \rightarrow M \ominus N / I, a \mapsto(a,-a)+I$. The image of $A$ is a $P$-invariant ideal in $M \ominus N / I$. It is readily checked that the precrossed module $\bar{\partial}$ induces a crossed module $\partial: M \ominus N /\{I+A\} \rightarrow P$.

The following proposition is a routine exercise.

Proposition 14. i) The above crossed module $\partial: M \ominus N /\{I+A\} \rightarrow P$ is isomorphic to the pushout crossed module $\partial: M \circ N \rightarrow P$.

ii) Suppose that the crossed modules $\partial_{L}: L \mapsto \stackrel{L}{P}, \partial_{M}: M \mapsto P, \partial_{N}: N \mapsto$ $P$ are all injective (that is, suppose $L, M, N$ are ideals in $P$ with $L \subseteq$ $M \cap N)$. Let $M^{\prime}=M / L$ and $N^{\prime}=N / L$. Then there is an isomorphism

$$
\operatorname{Ker}(\partial: M \underset{L}{\circ} N \rightarrow P) \cong \frac{M^{\prime} \cap N^{\prime}}{\left[M^{\prime}, N^{\prime}\right]} .
$$

\section{Applications to the homology of Lie algebras}

In this section suppose that the ground ring $\Lambda$ is a field. Let $G$ be a Lie algebra. We denote by $\mathbf{B}(G)$ or $\mathbf{K}(G, 0)$ the simplicial Lie algebra which in each dimension is equal to $G$, and in which each boundary and degeneracy map is the identity. Thus $\pi_{0}(\mathbf{K}(G, 0)) \cong$ and $\pi_{n}(\mathbf{K}(G, 0))=0$ for $n \geq 1$. The Eilenberg-MacLane homology of $G$ with coefficients in the ground ring $\Lambda$ can be defined (see [16, Section II6]) as

$$
H_{n}(G)=H_{n-1}(\mathbf{K}(G, 0)), \quad n \geq 1 .
$$

If $G$ is an abelian Lie algebra (that is, a $\Lambda$-module) then the trivial homomorphism $G \rightarrow 0$ can be considered as a crossed module. Let $\mathbf{K}(G, 1)$ denote the simplicial group equivalent to this crossed module under the correspondence of Theorem 2 . Thus $\pi_{1}(\mathbf{K}(G, 1)) \cong G$ and $\pi_{n}(\mathbf{K}(G, 1))=0$ for $n=0$ and $n \geq 2$. By analogy with [12] the appropriate homology for $G$ is possibly the homology of $\mathbf{K}(G, 1)$ (with a shift in dimension); we define

$$
H_{n}(G, 2) \cong H_{n-1}(\mathbf{K}(G, 1)), \quad n \geq 1 .
$$

The results of the preceding sections can be used to obtain information on $H_{n}(G)$ and $H_{n}(G, 2)$. Theorem 15 is new and analogous to group theoretic results given in [12]. Theorem 16 is an improvement on the exact sequence obtained in [9]; on taking $M=G$ it reduces to the well-known five term exact 
homology sequence [13]. The proof of Theorem 16 is modeled on techniques in [4].

THEOREM 15. For an abelian Lie algebra $G$ we have

$$
\begin{aligned}
& H_{1}(G, 2)=0, \\
& H_{2}(G, 2) \cong G, \\
& H_{3}(G, 2)=0, \\
& H_{4}(G, 2) \cong \Gamma(G) .
\end{aligned}
$$

Proof. The first two isomorphisms follow immediately from Theorem 8. The third and fourth isomorphisms follow immediately from Theorem 11 .

Theоrem 16. Let $G$ be a Lie algebra containing two ideals $M$ and $N$. (i) If $H_{2}(G /(M+N))=H_{3}(G /(M+N))=0$ (for instance if $\left.G=M+N\right)$ then there is an exact sequence

$$
\begin{aligned}
H_{2}(G) \rightarrow H_{2}(G / M) \oplus H_{2}(G / N) & \rightarrow \frac{M \cap N}{[M, N][M \cap N, G]} \rightarrow H_{1}(G) \\
& \rightarrow H_{1}(G / M) \oplus H_{1}(G / N) \\
& \rightarrow H_{1}(G /(M+N)) \rightarrow 0 .
\end{aligned}
$$

(ii) If on the other hand $M \cap N=[M, N]$ then there is an exact sequence

$$
\begin{aligned}
H_{2}(G) \rightarrow H_{2}(G / M) \oplus H_{2}(G / N) & \rightarrow H_{2}(G /(M+N)) \rightarrow H_{1}(G) \\
& \rightarrow H_{1}(G / M) \oplus H_{1}(G / N) \\
& \rightarrow H_{1}(G /(M+N)) \rightarrow 0 .
\end{aligned}
$$

Proof. There are canonical maps $\mathbf{B}(G) \rightarrow \mathbf{B}(G / M), \mathbf{B}(G) \rightarrow \mathbf{B}(G / N)$ which, working up to homotopy type, can be assumed to be free. We can also assume that $\mathbf{B}(G)$ is free. By Theorem 12 the pushout of simplicial Lie algebras

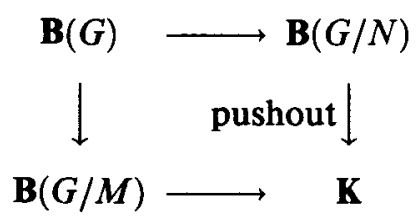

yields the exact sequence

$$
\begin{aligned}
H_{2}(G) \rightarrow H_{2}(G / M) \oplus H_{2}(G / N) & \rightarrow H_{1}(\mathbf{K}) \\
& \rightarrow H_{1}(G) \rightarrow H_{1}(G / M) \oplus H_{1}(G / N) \\
& \rightarrow H_{0}(\mathbf{K}) \rightarrow 0 .
\end{aligned}
$$

It remains to identify $H_{0}(\mathbf{K})$ and $H_{1}(\mathbf{K})$. 
Applying the functor $\chi$ of Section 5 to $(*)$ yields a pushout of crossed modules

$$
\begin{gathered}
(Q \hookrightarrow) \longrightarrow \quad(S \hookrightarrow F) \\
\downarrow \quad \text { pushout }\rfloor \\
(R \hookrightarrow F) \longrightarrow(R \circ S \rightarrow F) .
\end{gathered}
$$

Here $F$ is a free Lie algebra with ideals $Q, R, S$ satisfying $F / Q \cong Q$, $F / R \cong G / M, F / S \cong G / N$. We have $\pi_{0}(\mathbf{K}) \cong \operatorname{coker}(R \circ S \rightarrow F)$ and $\pi_{1}(\mathbf{K}) \cong \operatorname{ker}(R \circ S \rightarrow F)$. Thus $\pi_{0}(\mathbf{K}) \cong G /(M+N)$ and, by Proposition 14, $\pi_{1}(\mathbf{K}) \cong M \cap N /[M, N]$. Theorem 13 enables us to appropriately identify $H_{0}(\mathbf{K})$ and $H_{1}(\mathbf{K})$.

\section{Aspherical presentations}

In this section suppose that the ground ring $\Lambda$ is a field. Let $\mathscr{Z}$ be a set and $F$ the free Lie algebra on $\mathscr{Z}$. Let $\mathscr{R}$ be a set whose elements correspond to (not necessarily distinct) elements of $F$; for $r \in \mathscr{R}$ the corresponding element of $F$ is denoted $\xi r$. Let $R$ denote the ideal in $F$ generated by the set $\{\xi r: r \in \mathscr{R}\}$. Put $G=F / R$. Thus $\langle\mathscr{X} \mid \mathscr{R}\rangle$ is a presentation of the Lie algebra $G$.

From the set mapping $\xi: \mathscr{R} \rightarrow F$ we can construct the free precrossed module $\partial: P(\xi) \rightarrow F$, which is equivalent to a 1-truncated simplicial Lie algebra $P(\xi) \ominus F \stackrel{F}{\rightrightarrows} F$. Let $\mathbf{K}(\mathscr{X} \mid \mathscr{R})$ denote the 1-skeleton of $P(\xi) \ominus F \stackrel{\rightrightarrows}{\rightrightarrows} F$. Thus $\pi_{0} \mathbf{K}(\mathscr{X} \mid \mathscr{R}) \cong G$. We shall say that the presentation $\langle\mathscr{Z} \mid \mathscr{R}\rangle$ is aspherical if $\pi_{n} \mathbf{K}(\mathscr{X} \mid \mathscr{R})=0$ for $n \geq 1$.

If $\langle\mathscr{Z} \mid \mathscr{R}\rangle$ is aspherical then $\mathbf{K}(\mathscr{Z} \mid \mathscr{R})$ is weakly homotopic to $\mathbf{K}(G, 0)$. It follows from Theorem 10 that the Eilenberg-Maclane homology $H_{n}(G)$ is trivial for $n \geq 3$.

We can give a more manageable characterisation of aspherical presentations. Let $G^{e}$ be the universal enveloping algebra of $G$. Note that $R^{\mathrm{ab}}$ is a $G^{e}$-module in which $g \in G$ acts on $x+[R, R] \in R^{\text {ab }}$ by $g(x+[R, R])=$ $[\bar{g}, x]+[R, R]$ with $\bar{g}$ an element of $F$ representing $g$. Let $\oplus_{\mathscr{R}} G^{e}$ be the direct sum of copies of $G^{e}$, the copies indexed by $\mathscr{R}$; we think of $\oplus_{\mathscr{R}} G^{e}$ as the free $G^{e}$-module on the set $\mathscr{R}$. There is a unique surjective module homomorphism $\partial: \oplus_{\mathscr{R}} G^{e} \rightarrow R^{\mathrm{ab}}$ defined on basis elements $r \in \mathscr{R}$ by $\partial(r)=\xi r$.

THEOREM 17. The presentation $\langle\mathscr{X} \mid \mathscr{R}\rangle$ is aspherical if and only if $\partial: \oplus_{\mathscr{R}} G^{e} \rightarrow R^{\mathrm{ab}}$ is injective. 
Proof. Let $\widetilde{\mathbf{K}}(\mathscr{Z} \mid \mathscr{R})$ be the universal cover of $\mathbf{K}(\mathscr{X} \mid \mathscr{R})$. Thus

$$
\pi_{0} \widetilde{\mathbf{K}}(\mathscr{X} \mid \mathscr{R})=0
$$

and $\pi_{n} \widetilde{\mathbf{K}}(\mathscr{Z} \mid \mathscr{R}) \cong \pi \mathbf{K}(\mathscr{Z} \mid \mathscr{R})$ for $n \geq 1$. Theorem 8 implies $\pi_{1} \widetilde{\mathbf{K}}(\mathscr{Z} \mid \mathscr{R}) \cong$ $H_{1}(\widetilde{\mathbf{K}}(\mathscr{X} \mid \mathscr{R}))$. Let $\partial: C(\xi) \rightarrow F$ be the free crossed module on $\xi: \mathscr{R} \rightarrow$ $F$; this restricts to a crossed module $\partial: C(\xi) \rightarrow R$, which in turn induces a module homomorphism $\partial: C(\xi)^{\text {ab }} \rightarrow R^{\text {ab }}$. We have $H_{1}(\widetilde{\mathbf{K}}(\mathscr{X} \mid \mathscr{R})) \cong$ $\operatorname{ker}\left(\partial: C(\xi)^{\mathrm{ab}} \rightarrow R^{\mathrm{ab}}\right)$. Universal properties imply an isomorphism $C(\xi)^{\mathrm{ab}} \cong$ $\oplus_{\mathscr{R}} G^{e}$. It follows that $\pi_{1} \mathbf{K}(\mathscr{X} \mid \mathscr{R}) \cong \pi_{1} \widetilde{\mathbf{K}}(\mathscr{X} \mid \mathscr{R}) \cong \operatorname{ker}\left(\partial: \oplus_{\mathscr{R}} g^{e} \rightarrow R^{\mathrm{ab}}\right)$.

If $\partial: \oplus_{\mathscr{R}} G^{e} \rightarrow R^{\mathrm{ab}}$ is not injective then $\pi_{1} \mathbf{K}(\mathscr{X} \mid \mathscr{R}) \neq 0$ and so $\langle\mathscr{Z} \mid \mathscr{R}\rangle$ is not aspherical. If however $\partial: \oplus_{\mathscr{R}} G^{e} \rightarrow R^{\mathrm{ab}}$ is injective then $\pi_{1} \widetilde{\mathbf{K}}(\mathscr{X} \mid \mathscr{R})=0$; it follows from Theorems 8 and 10 that $\pi_{n} \mathbf{K}(\mathscr{X} \mid \mathscr{R}) \cong \pi_{n} \widetilde{\mathbf{K}}(\mathscr{X} \mid \mathscr{R})=0$ for $n \geq 1$, and thus that $\langle\mathscr{Z} \mid \mathscr{R}\rangle$ is aspherical.

Theorem 17 can be used to show that many one-relator presentations are aspherical. Consider for instance the Lie algebra $G$ with presentation $\langle x, y, z \mid[x,[y, z]]\rangle$. The proof of the injectivity of $\partial: G^{e} \rightarrow R^{\mathrm{ab}}, 1 \mapsto$ $[x,[y, z]][R, R]$ is left as an exercise. (This type of result is analogous to Lyndon's theorem asserting the asphericity of one-relator groups in which the relator is not a proper power [15].)

The following result might be of use in constructing aspherical presentations. It is analogous to the group-theoretic Theorem 3 in [20].

THEOREM 18. A presentation of the form $\langle\mathscr{Z} \mid \mathscr{R} \cup \mathscr{S}\rangle$ is aspherical if the presentations $\langle\mathscr{X} \mid \mathscr{R}\rangle$ and $\langle\mathscr{X} \mid \mathscr{S}\rangle$ are both aspherical and $R \cap S=[R, S]$. (Here $R$ and $S$ are the ideals in the free Lie algebra on $\mathscr{Z}$ generated by the elements corresponding to the sets $\mathscr{R}$ and $\mathscr{S}$.)

Proof. The canonical diagram of simplicial Lie algebras

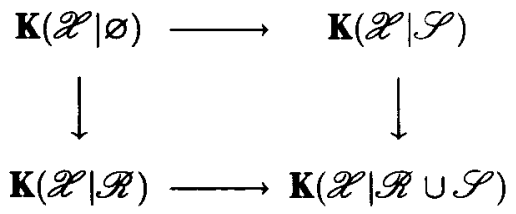

is a pushout. It follows from Proposition 14 that $\pi_{1} \mathbf{K}(\mathscr{X} \mid \mathscr{R} \cap \mathscr{S}) \cong R \cap$ $S /[R, S]$. Arguing as in the proof of Theorem 17 we see that if

$$
\pi_{1} \mathbf{K}(\mathscr{X} \mid \mathscr{R} \cap \mathscr{S}) \cong \pi_{1} \widetilde{\mathbf{K}}(\mathscr{X} \mid \mathscr{R} \cup \mathscr{R})=0
$$

then $\pi_{n} \mathbf{K}(\mathscr{X} \mid \mathscr{R} \cup \mathscr{S}) \cong \pi_{n} \widetilde{\mathbf{K}}(\mathscr{X} \mid \mathscr{R} \cup \mathscr{S})=0$ for $n \geq 1$. 


\section{References}

[1] H. J. Baues, Combinatorial homotopy and 4-dimensional complexes (Walter de Gruyter, Berlin, 1991).

[2] H. J. Baues and D. Conduché, 'The central series for Peiffer commutators in groups with operators', J. Algebra, 133 (1990), 1-34.

[3] K. S. Brown, Cohomology of groups, Graduate Texts in Math. 87 (Springer, New York, 1982).

[4] R. Brown, 'Coproducts of crossed $P$-modules: applications to second homotopy groups and to the homology of groups', Topology 23 (3) (1984), 337-345.

[5] D. Conduché, 'Modules croisés généralisés de Longueur 2', J. Pure Appl. Algebra 34 (1984), 155-178.

[6] E. B. Curtis, 'Simplicial theory', Advances in Math. 6 (1971), 107-209.

[7] A. Dold and D. Puppe, 'Homologie nicht-additiver Funktoren, Anwendungen', Ann. Inst. Fourier (Grenoble) 11 (1961) 201-312.

[8] J. Duskin, Simplicial methods and the interpretation of triple cohomology, Mem. Amer. Math. Soc. 163 (1975).

[9] G. J. Ellis, 'Nonabelian exterior product of Lie algebras and an exact sequence in the homology of Lie algebras', J. Pure Applied Algebra 46 (1987), 111-115.

[10] G. J. Ellis, ‘A nonabelian tensor product of Lie algebras', Glasgow. Math. J. 33 (1991), 101-120.

[11] G. J. Ellis, 'Homology of 2-types', J. London Math. Soc. (to appear).

[12] S. Eilenberg and S. MacLane, 'On the groups $H(\pi, n)$, II', Ann. of Math. (2) 60 (1954), 49-139.

[13] P. Hilton and U. Stammbach, $A$ course in homological algebra, Graduate Texts in Math. 4 (Springer, New York, 1971).

[14] C. Kassel and J.-L. Loday, 'Extensions centrales d'algèbres de Lie', Ann. Inst. Fourier (Grenoble) 33 (1982), 119-142.

[15] R. C. Lyndon, 'Cohomology theory of groups with a single defining relation', Ann. of Math. 52 (1950), 650-665.

[16] D. G. Quillen, Homotopical algebra, Lecture Notes in Math. 43 (Springer, New York, 1967).

[17] D. Simson and A. Tyc, 'Connected sequences of stable derived functors and their applications', Dissertationes Math. (Rozprawy Mat.) 111 (1974).

[18] E. Witt, 'Die Unterringe der freien Lieschen Ringe', Math. Zeitschrift 64 (1956), 195216.

[19] J. H. C. Whitehead, 'A certain exact sequence', Ann. of Math. 52 (1950), 51-110.

[20] M. A. Guttiérrez and J. G. Ratcliffe, 'On the second homotopy group', Quart. J. Math. Oxford Ser. (2) 32 (1981), 45-55.

\section{University College Galway}

National University of Ireland

Galway

Ireland 